\title{
The both-end readout system of the KOTO CsI calorimeter
}

\section{Nobuhiro Hara* for the KOTO collaboration}

Osaka University, Department of physics, Toyonaka, Osaka 560-0043, Japan

E-mail: haranobudchamp.hep.sci.osaka-u.ac.jp

The purpose of the J-PARC KOTO experiment is to observe the rare decay $K_{L} \rightarrow \pi^{0} v \bar{v}$. This decay directly violates the CP symmetry and is highly suppressed in the Standard Model $\left(\mathrm{BR}=3.0 \times 10^{-11}\right)$. This decay mode is thus sensitive to new physics that also breaks the $\mathrm{CP}$ symmetry. The signal of $K_{L} \rightarrow \pi^{0} v \bar{v}$ is only two photons with a finite transverse momentum. To detect this signal, we have an electromagnetic calorimeter made of CsI crystals. One of the main background sources is beam-halo neutrons producing two fake clusters in the calorimeter. To suppress the neutron background, we are preparing to attach 4096 Multi Pixel Photon Counters (MPPCs) on the upstream side of the crystals. We can distinguish neutrons and photons by measuring the timing difference between these MPPCs and PMTs attached on the downstream side of the crystals. For the both-end readout system, we developed front-end circuits to reduce the number of readout channel and to control the MPPCs.

XIV International Conference on Heavy Quarks and Leptons (HQL2018)

May 27- June 1, 2018

Yamagata Terrsa, Yamagata, Japan

\footnotetext{
*Speaker.
} 


\section{The KOTO experiment}

The aim of the J-PARC KOTO experiment is to search for new physics beyond the Standard Model (SM) by measuring the rare decay $K_{L} \rightarrow \pi^{0} v \bar{v}$. This decay is highly suppressed in the SM. The branching ratio of this decay was predicted to be $3.0 \times 10^{-11}$ in the SM [四].

The signal of $K_{L} \rightarrow \pi^{0} v \bar{v}$ is only two photons with a finite transverse momentum. To detect this signal, the KOTO detector consists of an electromagnetic calorimeter and hermetic veto counters as shown in Fig. $\mathbb{W}$. Most of detectors are in vacuum to minimize the amount of dead material such as a vacuum pipe between the decay vertex and the detectors. Figure $\square$ shows the KOTO calorimeter. The KOTO calorimeter is made of undoped cesium iodide (CsI) crystals. The central area of the calorimeter consists of 2240 small crystals $\left(25 \times 25 \times 500 \mathrm{~mm}^{3}\right)$, and the other area consists of 476 large crystals $\left(50 \times 50 \times 500 \mathrm{~mm}^{3}\right)$. The scintillation light from the crystal is detected with PMTs attached on the downstream side of the crystals. The waveforms of the signals from PMTs are recorded by 125-MHz 14-bit ADCs.

One of the main background sources in the data taken in 2013 []] was beam-halo neutrons. These neutrons produced two fake clusters in the calorimeter as shown in Fig. [1. To reach the sensitivity of the SM prediction, we need to suppress the neutron background by three orders of magnitude. We have already achieved a factor of hundred reduction based on the difference of the pulse shapes and the cluster shapes between neutrons and photons. To achieve another factor of ten reduction, we plan to upgrade the CsI calorimeter.

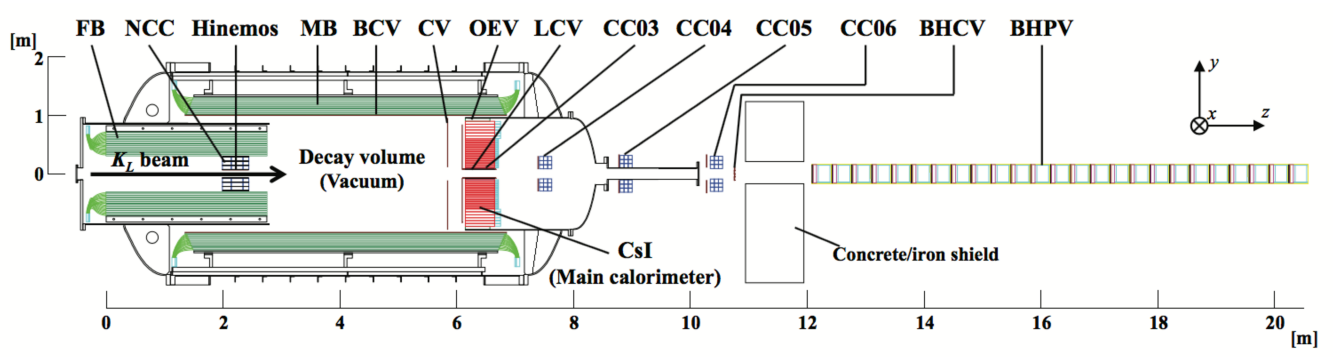

Figure 1: Sectional side view of the KOTO detector

\section{Both-end readout system}

To reject the neutron background, we will upgrade the CsI calorimeter with a "both-end readout system". Figure 4 shows the scheme of the both-end readout system. photons make electromagnetic showers in a shallow region of the CsI crystals. On the other hand, neutrons can make shower in a deeper region. We can thus distinguish neutrons and photons by measuring the depths of the interaction points. The depth information can be obtained from the difference of the arrival time of the scintillation light between the upstream and downstream surfaces of the crystals. In addition to the current PMT readout at the downstream, we will attach Multi Pixel Photon Counters (MPPCs), 


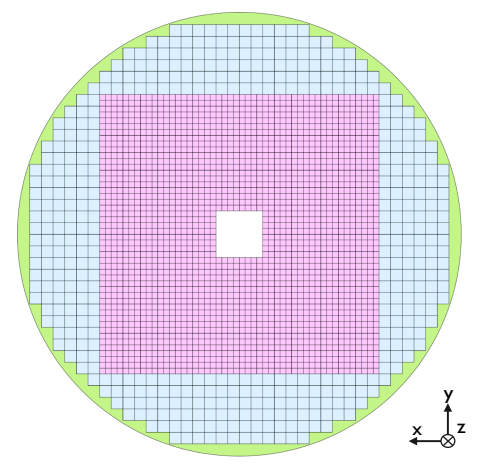

Figure 2: Sectional view of the CsI calorimeter

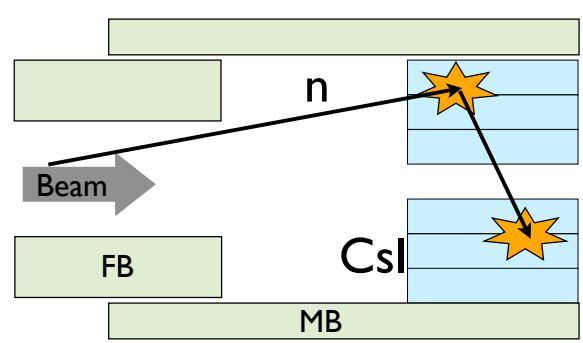

Figure 3: Scheme of the halo-neutron background event

Hamamatsu S13360-6050CS, on the upstream surface of the crystals. We will attach four MPPCs on each large crystal $\left(50 \times 50 \mathrm{~mm}^{2}\right)$, and one MPPC on each small crystal $\left(25 \times 25 \mathrm{~mm}^{2}\right)$. The total number of MPPCs is 4096.

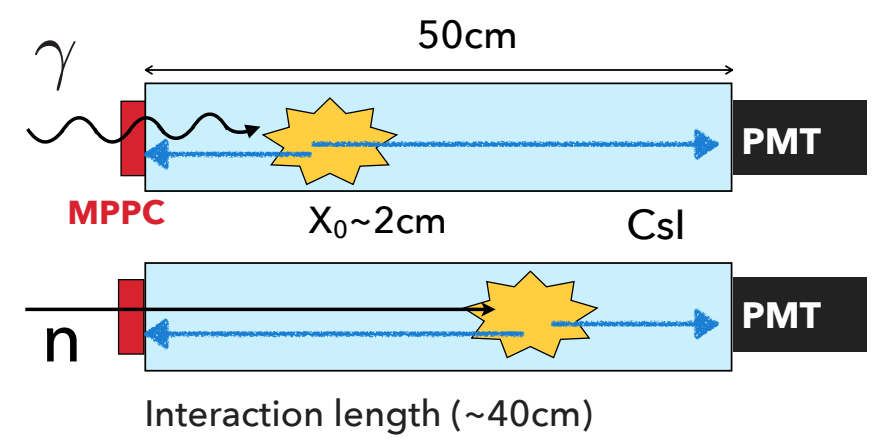

Figure 4: Concept of the both-end readout system

\section{Front-end circuit}

We will reduce the number of readout channels for 4096 MPPCs by using two methods, connecting MPPCs and summing up with an amplifier. We need a system to control and monitor the MPPCs and the readout circuits from outside of the vacuum because MPPCs and readout circuits are located inside of the vacuum tank. We describe the readout circuit and the controller in this section.

\subsection{Readout circuit including the MPPC connection}

First, four MPPCs are connected with a circuit as shown in Fig. [ to reduce the number of readout channels [B]. This circuit works as a series connection for the signal, and as a parallel 
connection for supplying bias voltage. This circuit makes the pulse shorter than connecting the MPPCs in parallel. Also, even if the dark current of MPPCs increases due to radiation damage, it can still supply fixed voltage to each MPPC. Second, the signals from four connected MPPCs are summed up with a summing amplifier shown in Fig. 6. By summing the signals from sixteen MPPCs, the number of readout channels is reduced from 4096 to 256. A group of 16 MPPCs covers a $100 \times 100 \mathrm{~mm}^{2}$ region of the calorimeter. The summed signals are converted to differential outputs, and they are read with $125-\mathrm{MHz} 14-$ bit ADCs.

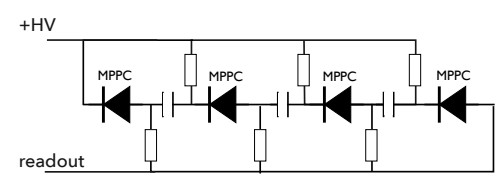

Figure 5: Scheme of the MPPC connection

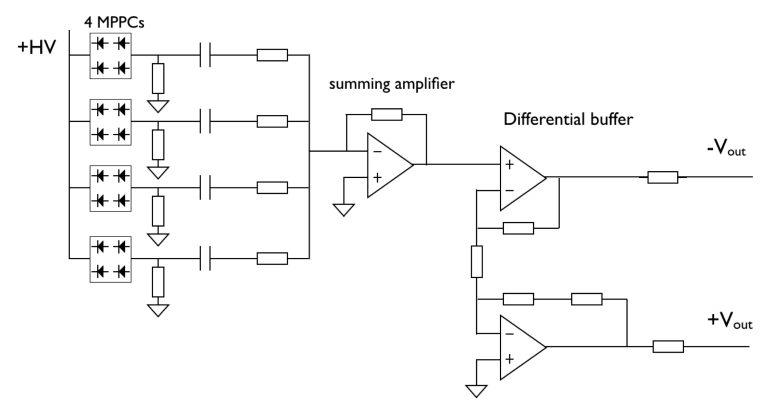

Figure 6: Circuit of the summing amplifier and the differential buffer

\subsection{Controller for MPPCs}

Figure $\square$ shows the schematics of the controller for MPPCs and readout circuits. The controller has several functions; it switches the bias voltage of each MPPC and monitors the bias voltage, temperature of the board, and the dark current of each MPPC. One controller board handles 32 MPPCs with similar operation bias voltage. Such common bias voltage is supplied to 32 MPPCs in parallel by a commercial voltage source with a remote control. Each MPPC can be switched off individually with a relay in our controller. The value of the bias voltage is recorded with a 16-bit ADC on the board. Temperature of the board is measured with a temperature sensor IC, and recorded with the same 16-bit ADC. Dark current of each MPPCs is amplified and recorded with another 16-bit ADC on the board.

Dark current of MPPC is typically $0.5 \mu \mathrm{A}$, but it increases by a factor hundred by a radiation damage after $3 \times 10^{7}$ seconds of operation with a $100 \mathrm{~kW}$ beam power. Thus monitoring the dark current with a wide dynamic range covering from $0.01 \mu \mathrm{A}$ to $100 \mu \mathrm{A}$ is necessary. The measurements with the control system are in good agreement with the measurements with a commercial digital multimeter as shown in Fig. 8 , which show I-V curves of MPPCs. We achieved measurements of the dark current from $0.01 \mu \mathrm{A}$ to $400 \mu \mathrm{A}$ with a precision of $1 \%$. Other functions also met the required performance.

\section{Conclusion}

The KOTO experiment studies the rare decay $K_{L} \rightarrow \pi^{0} v \bar{v}$ to discover new physics. We will upgrade the CsI calorimeter with the both-end readout system to reduce the halo-neutron background. 
The front-end circuits including the MPPC connection and the control system are developed. The number of readout channels is reduced from 4096 to 256 . The control system achieves the required performance in each function. In particular, we can measure the dark current from $0.01 \mu \mathrm{A}$ to $400 \mu \mathrm{A}$ with a precision of $1 \%$. We will install these systems in the summer of 2018 and will explore new physics with the upgraded CsI calorimeter from 2019.

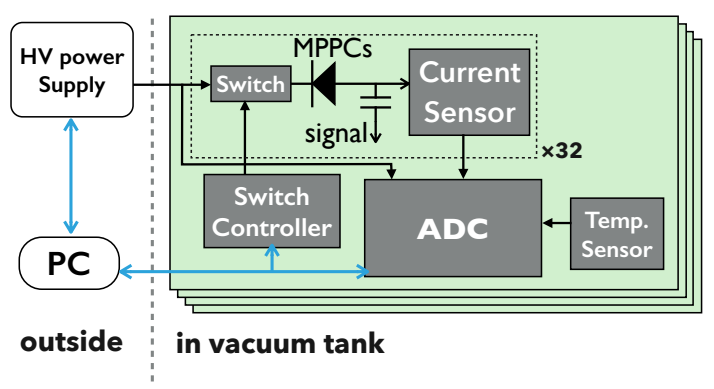

Figure 7: Schematics of the control system for MPPCs

(a) I-V curve of normal MPPC

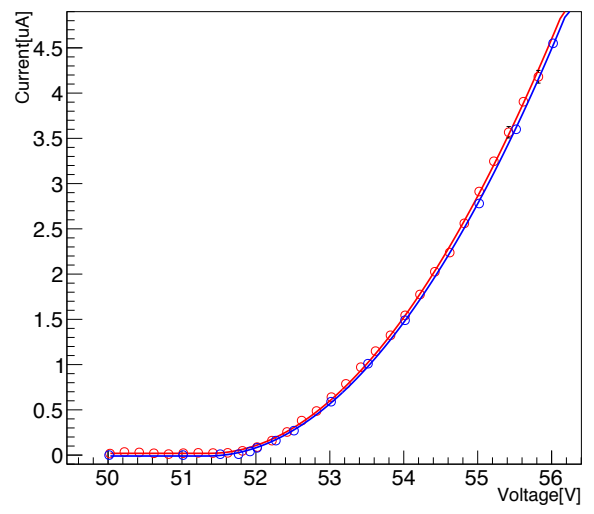

(b) I-V curve of irradiated MPPC

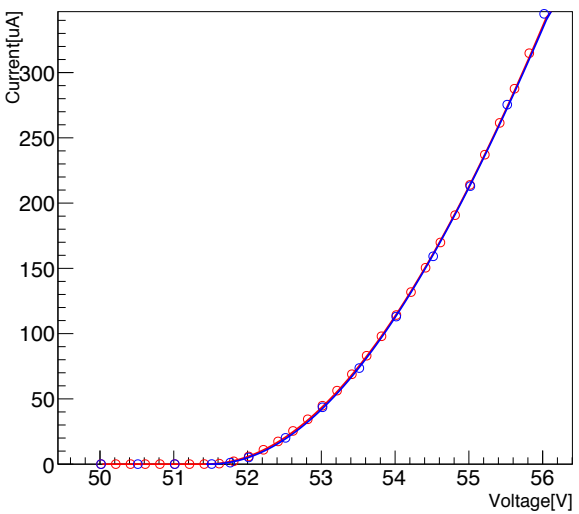

Figure 8: I-V curves of (a) normal MPPCs and (b) MPPCs with a dose equivalent to $3 \times 10^{7}$ seconds operation with $100 \mathrm{~kW}$ beam power. The red points show measurements with the control system and the blue points show measurements with a commercial digital multimeter.

\section{References}

[1] A. J. Buras, et al., $K^{+} \rightarrow \pi^{+} v \bar{v}$ and $K_{L} \rightarrow \pi^{0} v \bar{v}$ in the Standard Model: Status and Perspectives, JHEP 11 (2015) 033.

[2] J. K. Ahn, et al., A new search for the $K_{L} \rightarrow \pi^{0} v \bar{v}$ and $K_{L} \rightarrow \pi^{0} X^{0}$ decays, PTEP 2017 (2017) $021 \mathrm{C} 01$.

[3] M. Ogawa, 2016 master's thesis submitted to the University of Tokyo. 